\title{
Ueber das Bakankosin, ein durch Emulsin spaltbares Glykosid aus den Samen von Strychnos Vacacoua Baill.
}

\author{
Von E. B ourquelot und H. Hér is sey.
}

(Eingegangen den 24. XI. 1908.)

J. L a u re $\mathbf{t}^{\mathbf{3}}$ ) hat nach den Methoden, die der eine ron uns zum Nachweis des Rohrzuckers und der durch Emulsin spaltbaren Glykoside angegeben hat, Samen von fünf Strychnosarten untersucht und die Anwesenheit von Glykosiden in Strychnos Vacacoua Baill., Strychnos Nux vomica L. und Strychnos Ignatii B e r g. festgestellt.

Was die Samen von Strychnos Vacacoua $a^{2}$ ) anbetrifft, so war dies Ergebnis besonders interessant, da das Emulsin in einer Lösung

1) Recherche du saccharose et des glucosides dans quelques graines de la famille des Loganiacées, Journ. de Pharm. ot de Chim. [6], XXV., 225--228, 1907.

2) In der Arbeit von J. L a urent, wie auch in unseren ersten Mitteilungen über diesen Gegenstand (Journ. d. Pharm. et de Chim, [6], XXV., 417, 1907), wird Strychnos Vacacoua Baill. Strychnos Bakanko genannt. - Tatsächlich wurden die Samen, die L a u rent untersucht hatte, ihm durch Herrn Perrier de la B athie, der dieselben während seines Aufenthaltes auf Madagaskar gesammelt. hatte, als von einer Strychnosart, welche die Eingeborenen "Bakanko" nennen, und die botanisch noch nicht bestimmt war, stammend, übermittelt. Nur hatten wir damals bereits bemerkt, daß ,Bakanko" eine gewisse phonetische Aehnlichkeit mit "Vacacoua" hat, einem Namen, den Baillon als eine eigenartige Bezeichnung für einen Strychnos benutzte, der ebenso aus Madagaskar stammte - aber von einem mehr nördlichen Teil dieser Insel -, und von dem er nur eine unvollkommene Beschreibung geben konnte, da er keine reifen Früchte zur Verfügung hatte (Bull. soc. Linnéenne 242, 1880). Ende vorigen Jahres haben der Herr Prof. Jumelle und Herr Perrier de la $\mathbf{B}$ ath i e von neuem den Bakanko untersucht, so daß diese Art jetzt genau bekannt ist. R. J u melle schrieb uns zur Zeit folgendes darüber:

,Le Strychnos Bakanko est lo Str. Vacacoua B a i 1 l. et c'ast, en même temps, le Str. Gerrardi B r. H. (Bulletin de Kow, 1896) et, très probablement, le Str. Baroni Bath. de Madagascar. En tout cas, ce 
$(100 \mathrm{~g}$ in $100 \mathrm{cem})$ der noch nicht vollständig reifen Samen einen Drehungsumschlag nach rechts von $25^{\circ} 24^{\prime}$ hervorgerufen hatte $(1=2)$; diese hohe Zahl zeigte, daß sie eine große Menge Glykosides enthalten. So konnten wir auch hoffen, dieses Glykosid zu isolieren; das ist der Zweck, den wir verfolgten, nachdem $L$ a $u$ re $n t$ uns erklärt hatte, daß er diese Untersuchungen nicht fortsetzen wollte.

Tatsächlich sind unsere Versuche gelungen; wir haben reine Krystalle eines Glykosides erhalten, das wir Bakank os in genannt haben, in Rücksicht auf den Namen Bakanko, mit dem die Eingeborenen auf Madagaskar die Stammpflanze bezeichnen.

\section{Beschreibung der Früchte und der Samen von Strychnos Vacacoua.}

Die reifen Früchte von Strychnos Vacacoua sind beinahe rundliche Beeren, von orangegelber Farbe im frischen Zustande. Sie enthalten in der orangegelben Pulpa, die süß schmeckt und eßbar ist, 2-28 Samen. Diese sind von einer häutigen, gelben, zerreibbaren, leicht trennbaren Schale umhüllt; einzelne sind abgerundet, flach auf einer Seite, etwas gewölbt auf der anderen. aber die meisten sind unregelmäßig und erinnern im kleinen an die Samen aus den Ignatiusbohnen; in der Tat haben sie die Größe einer kleinen $\mathrm{NuB}$; ihr größter Durchmesser ist ungefähr $0,15 \mathrm{~m}$. Sie sind außerordentlich hart und zähe. Wenn man sie im destillierten Wasser aufweichen läßt, so quellen sie langsam auf, werden durchsichtig und lassen sich leicht mit dem Messer schneiden. Die Quellung ist bei gewöhnlicher Temperatur nach 4-5 Tagen vollständig. Der Embryo ist sehr klein, im Vergleich zu der Größe

qui est certain, e'est que la plante dont vous avez étudié les graines est l'espèce de $\mathrm{B}$ a i 11 o $\mathrm{n}$, que j'ai vue dans l'herbier du Muséum, en la comparant avec mes échantillons." Und einige Monate später, veroffentlichte or in Gemeinschaft mit Perrier de la Bathie eine Arbeit, in der er diese Anschauung bestätigte (Notes sur la flore du nord-ouest de Madagascar, Ann. du Musée colonial de Marseille, 15. année, 21. V. 1907).

Anderseits erhielten wir aus Majunga im Jahre 1907, eine beträchtliche Sendung reifer Früchte von Str. Vacacoua. P'er r jer de la Bathie, dem wir sie verdankten, teilte uns mit, was wir später vergleichsweise bestätigen konnten, da $\beta$ die ersten Samen die L a $u \mathrm{r}$ en t und wir selbst untersucht hatten, und die er auch die Giite gehabt hat uns zu senden, von Früchten, die $n$ ich vollständig reif waren, stammten. 
der Samen, die fast vollständig aus einem körnigen Albumen bestehen; dieser Embryo erinnert vollkommen an diejenigen der Strychnos Nux vomica und Strychnos Ignatii. 100 nicht vollständig reife Samen von denen, die $\mathrm{L}$ a $\mathrm{u}$ r e $\mathrm{n} \mathrm{t}$ und wir zu unseren ersten Cntersuchungen benutzt haben, wogen, an der Luft getrocknet und von ihrer Schale befreit, $73,5 \mathrm{~g}$, was für jeden einzelnen Samen. im Mittel, $0,735 \mathrm{~g}$ ausmacht. 100 reife, lufttrockene Samen von denen, die P e r i e r d e la Bath i e uns aus Majunga gesehickt hatte, wogen $87,5 \mathrm{~g}$, was für einen einzelnen Samen, im Mittel. $0,875 \mathrm{~g}$ ausmacht; jede Sehale wog im Mittel $0,195 \mathrm{~g}$.

\section{Nachweis eines durch Emulsin spaltbaren Glykosides in den vollständig reifen Samen.}

J. La u rent hatte seine Untersuchungen, wie wir schon oben gesagt haben, mit Samen, die noch nicht ganz reif waren, angestellt, deshalb wollten wir uns überzeugen, ob in den vollständig reifen Samen ein Glykosid noch vorhanden sei; bekanntlich verschwinden oder umwandeln sich ja bei vielen Pflanzen während des Trocknens die wirksamen Bestandteile der Früchtc wie der Samen.

Zunächst wiederholten wir mit den reifen Samen unsere Versuche mit Hilfe des Invertins und des Emulsins. $50 \mathrm{~g}$ bei $28^{\circ}$ getrocknetes Pulver von reifen Samen wurden mit kochendem Alkohol von $90^{\circ}$ behandelt, die alkoholische Lösung im luftverdünnten Raume abdestilliert und der Rückstand mit Thymolwasser zu $100 \mathrm{ccm}$ aufgenommen. Die auf diese Weise erhaltene Lösung zeigte eine Linksdrehung $\left(-7^{0} 48^{\prime}\right)$ im $2 \mathrm{dm}$-Rohr und enthielt $0,727 \mathrm{~g}$ reduzierenden Zuckers (auf Glykose berechnet). Jie Flüssigkeit wurde dann mit starkem Alkohol getötetem und trockenem Oberhefepulver versetzt ( $\mathrm{g}$ g Hefepulver zu $100 \mathrm{ccm}$ Flüssigkeit) und im Trockenschrank bei $28^{\circ}$ gestellt.

Nach vier Tagen, nachdem die Wirkung des Invertins beendet war, ergab die polarimetrische Prüfung $\alpha=-8^{\circ} 24^{\prime}(1=2)$, während die Menge des reduzierenden Zuckers auf $1,142 \mathrm{~g}$ in $100 \mathrm{ccm}$ gestiegen war. Es hatte somit nach der Einwirkung von Invertin eine Neubildung von $0,415 \mathrm{~g}$ reduzierenden Zuckers stattgefunden. Dieses entspricht $0,393 \mathrm{~g}$ Rohrzucker in $100 \mathrm{ccm}$ der Lösung, d. h. $0,786 \mathrm{~g}$ Rohrzucker für $100 \mathrm{~g}$ Samen.

Die übrige Flüssigkeit wurde auf $100^{\circ}$ gebracht, nach dem Erkalten mit 0,30 g Emulsin versetzt und bei $28^{\circ} \mathrm{im}$ Trockenschrank stehen gelassen. 
Dit Beobachtung im Polarimeter ergab folgende Daten:

Dauer der Emulsinwirkung.

$$
\begin{aligned}
& 0 \\
& 4 \text { Tage } \\
& 14 \text { Tage } \\
& 78 \text { Tage }
\end{aligned}
$$

Drehung $(1=2)$.

$$
\begin{array}{r}
-8^{0} 24^{\prime} \\
-6^{0} 58^{\prime} \\
-5^{0} 34^{\prime} \\
+1^{0} 26^{\prime}
\end{array}
$$

Aus diesen Versuchen ersieht man, daß die rejfen Samen von Bakanko ein durch Emulsin spaltbares Glykosid enthalten, und daß die Hydrolyse dieses Glykosides durch das Ferment sich außerordentlich langsam vollzieht.

Im Laufe der nachstehenden Untersuchungen hat es sich herausgestellt, daß das Glykosid, das wir aus den reifen Samen erhalten haben, identisch mit dem aus den nicht vollständig reifen Samen ist.

Das Bakankosin wird aus diesen beiden Sorten Samen nach demselben Extraktionsverfahren hergestellt.

\section{Darstellung des Bakankosins.}

Man kann das Bankakosin auf zwei verschiedene Weisen erhalten; in beiden Fällen verfährt man mit den trockenen Samen, die, befreit von ihrer Schale, halb fein gemahlen und mit Aether erschöpft waren - um die kleine Menge des Fettes, die sie enthalten, zu entfernen -., wie folgt:

I. Das entfettete Pulver wird am Rückflußkühler mit Essigäther (1000 ccm für $100 \mathrm{~g}$ Pulver) 30 Minuten lang gekocht. Man filtriert heiß in einem großen Kolben und läßt 24 Stunden stehen. Es setzt sich hierdurch an den Wänden des Kolbens ein leichter, farbloser, krystallinischer Niederschlag ab.

Die Flüssigkeit wird von den Krystallen abgegossen und alsdaun von neuem mit dem extrahierten Pulver in Berührung gebracht; man läßt sie noch 30 Minuten lang kochen und filtriert dann heiß in demselben Kolben; diese Operation wird noch zweimal, nach je 24 Stunden Zwischenzeit, wiederholt. Endlich wird der ausgeschiedene Niederschlag sorgfältig abgesaugt und hierauf mit aiedendem Alkohol (20 ccm für $100 \mathrm{~g}$ ursprüngliches Pulver) am Rückflußkühler aufgenommen. Man filtriert heiß. Es tritt alsdann eine Krystallisation ein, die nach 3-4 Tagen vollständig beendet ist.

Die abgesaugten farblosen Krystalle stellen das Glykosid in verhältnismäßig sehr reinem Zustande dar. Man erhält auf 
diese Weise etwa $1 \mathrm{~g}$ Krystalle für $100 \mathrm{~g}$ Pulver; dieselben werden schließlich sukzessive dreimal aus Alkohol von $95^{\circ}$ und endlich aus heißem Wasser (4 ccm für $1 \mathrm{~g}$ ) umkrystallisiert.

Dieses Verfahren gibt zwar ein sicheres Resultat, jedoch würde man dabei eine ungeheure Menge des Lösungsmittels verbrauchen, wollte man das Pulver vollständig erschöpfen, da das Glykosid außerordentlich schwer löslich in Essigäther ist.

II. Man extrahiert das entfettete Pulver mit heißem Alkohol von $95^{\circ}$ am Rückflußkühler.

Die alkoholische Lösung wird bei Gegenwart von etwas Calciumkarbonat unter vermindertem Druck zur Trockne abdestilliert. Man nimmt den Rückstand mit Wasser auf, filtriert und versetzt die Flüssigkeit mit etwas Oberhefe, um den Rohrzucker zu vernichten. Nach 24 Stunden filtriert man von neuem und dampft bis zur Sirupkonsistenz ein; das Bakankosin krystallisiert dann in großen, gefärbten Krystallen aus, die abgesaugt werden und zur weiteren Reinigung, zunächst aus Wasser (4 ccm für $1 \mathrm{~g}$ ), unter Zusatz von Tierkohle, dann aus Alkohol von $95^{\circ}$ ( $1 \mathrm{cem}$ für $1 \mathrm{~g}$ ) und sehließlich von neuem aus Wasser umkrystallisiert werden.

Aus Wasser scheidet sich das Bakankosin in großen, schweren Krystallen aus, die an den Wänden des Kolbens anhaften, während es aus heißem Alkohol von $95^{\circ}(10 \mathrm{ccm}$ für $1 \mathrm{~g}$ ) in filzigen Nadeln, die die ganze Masse des Lösungsmittels anfüllen, krystallisiert.

Die nachstehenden Eigenschaften beziehen sich auf die aus. Wasser erhaltene Verbindung.

Die noch nicht vollständig reifen Samen geben eine größere Ausbeute an Glykosid als die reifen. Als man gleichzeitig nach der Extraktionsmethode mit Alkohol einerseits $760 \mathrm{~g}$ entfettetes Pulver reifer Samen und andererseits $550 \mathrm{~g}$ entfettetes Pulver nicht vollständig reifer Samen behandelte, ergab sich im ersten Falle $7 \mathrm{~g}$ krystallisiertes Rohbakankosin, d. h. eine Ausbeute von $0,92 \%$, wogegen im zweiten Falle $20 \mathrm{~g}$ krystallisiertes Rohbakankosin bezw. eine Ausbeute von 3,6\% erhalten wurden. Der Gehalt der Samen an Bakankosin nimmt somit mit dem Reifen derselben ab.

Es soll noch bemerkt werden, daß, wenn man die Drehungen der extraktiven Flüssigkeiten vergleicht, die einerseits aus den grünen und anderseits aus den reifen Samen hergestellt wurden, so geben dieselben, obwohl ihre Drehungen stark voneinander abweichen (nach L a u rent $-13^{\circ} 12^{\prime}$ für die grünen und $7^{\circ} 48^{\prime}$ für die reifen Samen), keinen Aufschluß über die beträchtliche Verschiedenheit, die bei der Ausbeute an Glykosid beobachtet wird. Vielleicht hat sich ein Teil des Bakankosins der grünen Samen 
während des Reifens derselben in einen anderen Bestandteil umgewandelt und trotzdem die ursprüngliche Eigenschaft beibehalten stark nach links die Polarisationsebene zu drehen.

Wir haben auch nach der Emulsinmethode die Schale (Episperm) der Samen und die Pulpa untersucht. Weder die eine noch die andere enthalten jedoch ein durch Emulsin spaltbares Glykosid.

Die Schale ist sehr reich an reduzierendem Zucker.

\section{Eigenschatten des Bakankosins.}

Das aus Wasser krystallisierte Bakankosin erscheint in großen

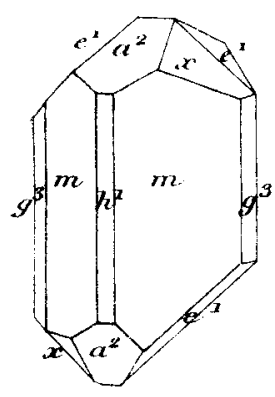

Krystallen, die sehr beständig an der Luft, farblos und geruchlos sind.

Herr $W$ y $r$ o $u$ b of $f$, dem wir unseren verbindlichsten Dank aussprechen, war so liebenswürdig, das aus Wasser krystallisierte Bakankosin in krystallographischer Hinsicht zu untersuchen. Wir geben hier seine Beschreibung an:

,Les cristaux appartiennent à l'hémiédrie holoaxe de système orthorhombique; les faces $x$ (122) forment un tétraèdre comme dans le sulfate de magnésie.

Faces observées: $h^{1}(100), m(110), g^{3}(120), e^{1}(011), a^{2}(102), x(122)$.

La forme est très voisine de celle d'un cube rapporté à deux axes binaires et un axe quaternaire. On a en effet:

$$
0,7089: 1: 0,9897
$$

Angles

$m m(\overline{\overline{11}} 0 \quad 110)$

$g^{3} g^{3}(\overline{120} 120)$

$a^{2} a^{2}(\overline{102} 102)$

$e^{1} e^{1}(\overline{01} 1011)$

$e^{1} a^{2}$ (011 102)

$e^{1} m(011 \quad 110)$

$x a^{2} \quad\left(\begin{array}{ll}122 & 102\end{array}\right)$

$\left.\begin{array}{lll}x g^{3} & (122 & 120\end{array}\right)$

$x h^{1}(1.22100)$

xm (122 110)
Calculés Mesurés

$\begin{array}{cr}- & - \\ - & * 109^{0} 20^{\prime} \\ 70^{0} 24^{\prime} & 70^{0} 28^{\prime} \\ - & * 110^{0} 10^{\prime} \\ 90^{\circ} 36^{\prime} & - \\ 125^{\circ} 33^{\prime} & 125^{0} 36^{\prime} \\ 114^{0} & 113^{\circ} 54^{\prime} \\ 140^{\circ} 56^{\prime} & 140^{0} 55^{\prime} \\ 140^{\circ} 27^{\prime} & 140^{\circ} 20^{\prime} \\ 116^{\circ} 23^{\prime} & 116^{\circ} 24^{\prime} \\ 136^{\circ} 38^{\prime} & 136^{\circ} 40^{\prime}\end{array}$

Les cristeux assez troubles et extrêmement fragiles ne se prêtent pas à la détermination des propriétés optiques." 
Das lufttrockene Bakankosin hat einen bitteren Geschmack: es schmilzt zunächst scharf bei $157^{\circ}$, wird wieder fest und schmilzt von neuem, doch unvollständig bei etwa $200^{\circ}$.

Es ist ziemlich leicht löslich in kaltem, aber viel löslicher in heißem Wasser und in Alkohol, schwer löslich dagegen in Essigäther und fast unlöslich in Aether.

Wir haben seine Auflösbarkeit bei $20^{\circ}$ in Wasser, Aethylalkohol, Methylalkohol und Essigäther ermittelt und folgende Resultate erhalten:

$100 \mathrm{~g}$ destilliertes Wasser lösen $8,053 \mathrm{~g}$ wasserfreies Bakankosin.

100, , Aethylalkohol v. $95^{\circ},, \quad 1,794$,

100 , Methylalkohol ", 24,500,,

100 ,, wasserfr. Essigäther , $0,032,$, oder 1 Teil Bakankosin löst sich bei $20^{\circ} \mathrm{in}$

3164,5 Teilen wasserfreien Essigäther

$55,7, \quad$ Aethylalkohol von $95^{\circ}$

12,4 , destillierten Wasser

4,08 ,. Methylalkohol.

An der Luft, bis zum konstanten Gewicht getrocknet, vel'liert es im Mittel 4,80\% Wasser, wenn man es auf $115-120^{\circ}$ erhitzt.

1. Produkt, welches aus nicht vollständig reifen Samen mit. Fssigäther erhalten wurde:

$0,9479 \mathrm{~g}$ verloren $0,0450 \mathrm{~g}$ bezw. $4,74 \%$.

2. Produkt, welches aus nicht vollständig reifen Samen wit Alkohol von $95^{\circ}$ erhalten wurde:

$2,0225 \mathrm{~g}$ verloren 0,0988 , d. h. $4,88 \%$.

3. Produkt, welches aus reifen Samen mit Alkohol von $9.5^{\circ}$ erhalten wurde:

$$
1,0193 \mathrm{~g} \text { verloren } 0,0489 \text { bezw. } 4,79^{\circ} \text {. }
$$

Ueber Schwefelsäure verliert das Bakankosin nicht merklich Wasser; der Gewichtsverlust erfolgt sehr langsam und unvollständig im Vakuum über Schwefelsäure sowie im Wassertrockenschranke.

Beim Liegen an der Luft nimmt das getrocknete Produkt in 2-3 Tagen das verlorene Wasser wieder auf.

Das Bakankosin ist linksdrehend.

Die lufttrockenen Krystalle ergaben:

1. Produkt, welches aus unreifen Samen mit Essigäther halten wurde:

$\sigma .[\mathrm{D}]=-195^{\circ}, 2\left(\mathrm{v}=25,1=2, \mathrm{p}=0,5443, \quad \alpha=-8^{0} 30^{\prime}=-8^{0}, 50\right)$.

2. Produkt, welches aus unreifen Samen mit Alkohol von $95^{0}$ erhalten wurde:

$\left.x_{[\mathrm{l}}\right]=-195^{\circ}, 6\left(\mathrm{v}=25,1=2, \mathrm{p}=0,4794, \alpha=-7^{\circ} 30^{\prime}=-7^{\circ}, 50\right)$. 
3. Produkt, welches aus reifen Samen mit Alkohol von $95^{\circ} \mathrm{er}$ halten wurde:

$$
\text { \% }[\mathrm{v}]=-196^{\circ}, 8\left(\mathrm{v}=15, \mathrm{l}=2, \mathrm{p}=0,508, \alpha=-13^{\circ} 20^{\prime}=-13,333\right) \text {. }
$$

Durch kleine Mengen von Barytwasser ( $\mathrm{N} / 100^{-}$und $\mathrm{N} / 10^{-}$-Daues 24 Stunden) erleidet das Bakankosin keine molekulare Umlagerung im Gegensatz zu anderen stickstoffhaltigen Glykosiden, wie Amygdalin, Amygdonitrilglykosid und Sambunigrin ${ }^{\mathfrak{1}}$ ).

Das Bakankosin liefert keine charakteristischen Farbreaktionen beim Behandeln mit Schwefel-, Salpeter- oder Salzsäure, kalt oder beim Erhitzen. Mit heißer Schwefelsäure gibt es eine schwache rotbraune Färbung, die aber keineswegs spezifisch ist.

Das Bakankosin hinterläBt keine Asche. Es ist stickstoffhaltig, da sich einerseits alkalische Dämpfe entwickeln, wenn man es in Gegenwart von Natronkalk erhitzt, und andererseits, da eine Cyanverbindung entsteht, die man leicht durch die Berliner. blaureaktion charakterisieren kann, wenn man ein Gemisch ron Bakankosin und Natrium erwärmt.

Durch verdünnte kochende Säuren wird das Bakankosin langsam gespalten; so war in einer Lösung, die $3 \mathrm{~g}$ des Glykosides und $3 \mathrm{~g}$ Schwefelsäure in $100 \mathrm{ccm}$ enthielt, die Spaltung erst nach Verlauf von 6-7 stündigem Kochen vollendet.

Als unter diesen Bedingungen $4,50 \mathrm{~g}$ der Verbindung gespalten wurden, resultierte, nachdem die hydrolysierte Flüssigkeit mit Calciumkarbonat neutralisiert, alsdann verdampft und des Rückstand mit Alkohol von $95^{\circ}$ wieder aufgenommen wurde, eine kleine Menge eines krystallisierten Spaltungsproduktes, welche nach dem Umkrystallisieren aus Alkohol von $95^{\circ}$ mit d - G l y k o s e identifiziert werden konnte (Schmp. $146-147^{\circ}, \wedge_{[0}=+51^{\circ} .9$ ).

Das Emulsin wirkt ebenfalls nur sehr langsam auf das Bakankosin ein; in einer Lösung, die in $100 \mathrm{ccm} 2 \mathrm{~g}$ Bakankosin und 0,5 g Emulsin enthielt, war die Spaltung erst nach Verlauf von 7 Wochen vollständig $\left(t=18-20^{\circ}\right)$. Diese Versuche wurden mehrmals mit Emulsinen verschiedener Herkunft angestellt und haben immer zu demselben Resultat geführt.

Die Fermente von Aspergillus niger üben auch eine hydrolysierende, jedoch sehr langsame Wirkung auf das Bakankosin aus.

Wir sehen also, daß das Bakankosin wie alle anderen durch Emulsin spaltbaren Glykoside linksdrehend ist, und daB es bei der Spaltung d-Glykose liefert.

1) B ourqelot et $H$ érissey, Journal d. Pharm. et de Chimie (6), XXVI., p. 5, 1907. 
Die kryoskopische Molekularbestimmung des Bakankosins ergab in wässeriger Lösung:

$$
\mathrm{M}=18,5 \times \frac{3,786}{0,195}=359,
$$

Wasser 24,35 g, wasserfreies Bakankosin 0,923, $\mathrm{A}=0^{0}, 195$.

Die Elementaranalyse der krystallisierten lufttrockenen Verbindung ergab folgende Werte:

1. $0,2585 \mathrm{~g}$ lieferten $0,4834 \mathrm{~g} \mathrm{CO}_{2}$ und $0,1576 \mathrm{~g} \mathrm{H}_{2} \mathrm{O}$.

2. $0,2359 \mathrm{~g}$ lieferten $0,4433 \mathrm{~g} \mathrm{CO}_{2}$ und $0,1465 \mathrm{~g} \mathrm{H}_{2} \mathrm{O}$.

3. $0,4545 \mathrm{~g}$ lieforten $0,01653 \mathrm{~g} \mathrm{~N}$.

Diese Zahlen zeigen, im Verein mit den vorher für den Gewichtsverlust ermittelten Daten, daß das Bakankosin ein Molekül Krystallwasser enthält, und daß ihm somit die Formel

$$
\mathrm{C}_{16} \mathrm{H}_{23} \mathrm{O}_{8} \mathrm{~N}+\mathrm{H}_{2} \mathrm{O}
$$

zukommt, was sich auch aus den folgenden Werten ergibt:

Berechnet für $\mathrm{C}_{16} \mathrm{H}_{23} \mathrm{O}_{8} \mathrm{~N}+\mathrm{H}_{2} \mathrm{O}$ :

Molekulargewicht . . $357+18$

Krystallwasser . . $\quad 4,80$ p. 100

C . . . . . . . . 51,20

H. . . . . . . 6,66

N. . . . . . . 3,73
Gefunden:

359 f. d. wasserfr. Verbindung

$\begin{array}{rrr}\mathbf{4}, 74 & \mathbf{4 , 8 8} & \mathbf{4 , 7 9} \\ 51,00 & 51,24 & \\ 6,77 & 6,89 & \\ 3,63 & . & \end{array}$

Was die Gleichung betrifft, welche die Einwirkung des Emulsins oder der verdünnten Schwefelsäure auf das Bakankosin veranschaulicht, so wird man dieselbe erst dann mit Sicherheit aufstellen können, wenn man den Charakter des oder der Spaltungsprodukte, welche die Glykose begleiten, kennen wird. Vorläufig wird man sie, da sich wahrscheinlich nur ein Molekül Traubenzucker bildet, in folgender Weise ausdrücken können:

$$
\mathrm{C}_{16} \mathrm{H}_{23} \mathrm{O}_{8} \mathrm{~N}+\mathrm{H}_{2} \mathrm{O}=\mathrm{C}_{6} \mathrm{H}_{12} \mathrm{O}_{66}+\mathrm{C}_{10} \mathrm{H}_{13} \mathrm{O}_{3} \mathrm{~N} \text { (?) }
$$

Wir haben noch die eventuelle Gegenwart des Bakankosins in der BrechnuB untersucht, da, wie wir oben gesehen haben, dieselbe ein durch Emulsin spaltbares Glykosid enthält, jedoch ergab sich. hierbei ein negatives Resultat. Wir können aber, wegen der Schwierigkeiten, mit welchen diese Untersuchungen der Brechnuß verknüpft sind, namentlich, da sie reich an anderen wirksamen Stoffen ist, noch nicht unbedingt auf die Abwesenheit des Bakankosins in derselben schließen.

Wir haben auch prüfen wollen, ob das Bakankosin giftig für Tiere ist. Dem Meerschweinchen unter die Haut eingespritzt $(0,28 \mathrm{~g}$ pro $\mathrm{kg}$ des Tieres), hat es keine unangenehmen Zufälle hervorgerufen. Auch mit Lösungen, denen man lange Zeit vorher etwas Emulsin zugefügt hatte, um die Spaltungsprodukte wirken zu lassen, wurden keine Störungen beobachtet. 